\title{
Delayed matching-to-sample in rats in a Y-maze: Instances of facilitation and immediate cross-modal transfer
}

\author{
M. RAY DENNY, CARLA CLOS, and MARK RILLING \\ Michigan State University, East Lansing, Michigan
}

\begin{abstract}
Of 6 rats initially trained on matching-to-sample (MTS) with black/white stimuli, 3 learned the problem and, on delayed matching, produced a stable forgetting function. One rat that failed with black/white stimuli learned hardware cloth/smooth floor MTS. Two of the 4 learners showed immediate transfer of matching to an alternate stimulus dimension. All rats that learned were trained with a differential outcome condition that was shifted to equal outcomes after clear preference for superior outcome had developed, that is, after they attended to the appropriate dimension. All rats under this condition learned in fewer than $\mathbf{7 0 0}$ trials.
\end{abstract}

There have been only a few reports of successful delayed matching-to-sample or its variants using rats as subjects (Cohen, Escott, \& Ricciardi, 1984; Cohen, Galgan, \& Fuerst, 1986; Heise \& Hudson, 1985; Spencer, Pontecorvo, \& Heise, 1985; Wallace, Steinert, Scobie, $\&$ Spear, 1980), and presumably there has been only one matching-to-sample (MTS) study using rats that did not use an automated operant procedure (Lashley, 1938). Lashley used the jumping stand and had no success in training rats to match to sample; however, he administered only 200 trials in this aborted effort. With operant procedures, subjects typically require a thousand or more trials before learning MTS.

In the present study of short-term memory in the rat, we also did not employ an automated procedure. Instead, we presented the sample stimuli along the stem and presented the comparison stimuli in the arms of a Y-maze. In addition, we addressed the question as to whether the use of a differential outcome (DO) procedure (G. B. Peterson \& Trapold, 1980; Trapold, 1970) would facilitate the learning of MTS, that is, whether it would yield the customary DO effect for conditional discrimination learning in rats. With the procedures of the present study, we had definite reservations about how effective DO would be. Since in our MTS task, the subject had to learn half the time to directly approach the color of the arm associated with a smaller reinforcer (a possible source of aversive frustration by comparison with the larger reinforcer), this could lead to a consistent preference for the color associated with the larger reinforcer (i.e., roughly $50 \%$ correct performance). In the usual conditional discrimination, however, the subject at the choice point simply turns (presses) either left or right and neither avoids

This research was supported in part by Grant BNS-8605524 from the National Science Foundation. Requests for reprints may be sent to M. Ray Denny, Department of Psychology, Michigan State University, East Lansing, MI 48824-1117. nor approaches external cues that have been paired with reinforcers.

Finally, since the study involved the use of two different sets of MTS stimuli, we investigated the possibility of transfer of a matching rule from one problem to the other.

\section{METHOD}

\section{Subjects}

Six Holtzman-derived albino males that were approximately 180 days old served as subjects. They were gentled for 10 days while being reduced to $80 \%$ of their free-feeding weights.

\section{Apparatus}

The experimental device was an elevated Y-maze with the choice arms $90^{\circ}$ apart. The stem was $68 \mathrm{~cm}$ long $\times 6.3 \mathrm{~cm}$ wide, the choice point section was $8.8 \mathrm{~cm}$ wide $\times 2.2 \mathrm{~cm}$ long, and the arms were $36.3 \mathrm{~cm}$ long $\times 10 \mathrm{~cm}$ wide with a small recess in the floor at the end of each arm serving as a food dish. The whole maze was painted an intermediate gray. In addition, there were three black hurdles and three white hurdles, $3.9 \mathrm{~cm}$ high $\times 3.7 \mathrm{~cm}$ thick, plus cardboard floor inserts that were black on one side and white on the other. One insert covered the stem (sample); the other two inserts together with the hurdles cued the two arms (comparison), each arm being different from the other and half the time white and half the time black. Two black or two white hurdles were always in the stem, matched to the color of the floor; they were located $27 \mathrm{~cm}$ and $50 \mathrm{~cm}$ from the start end of the stem. The floor inserts for the arms extended $9 \mathrm{~cm}$ from each arm-hurdle into the gray choice point area and $20 \mathrm{~cm}$ past the hurdle into the arm, leaving only the goal section of each arm gray.

Since the wall directly behind the maze was off-white, a patch of black cloth $(18 \times 32 \mathrm{~cm})$ was taped to the wall directly behind the arm that was black for that trial. A shield (black on one side, white on the other) that was $23 \mathrm{~cm}$ high $\times 44 \mathrm{~cm}$ wide prevented a rat from seeing any stimuli related to the arms until it arrived at the choice point.

During delay trials (memory testing), the black cloth on the wall and the black/white shield were dispensed with. Instead, a solid gray shield, $23 \mathrm{~cm}$ high $\times 30 \mathrm{~cm}$ wide, was placed in the choice point region, so that after crossing the second hurdle, the rat was in a neutral gray area $28 \mathrm{~cm}$ long, where it spent the delay (the stimuli in the arms were not visible).

When cues were shifted from color to tactual (hardware cloth vs. smooth floor), the gray stem was covered with gray hardware cloth for $53 \mathrm{~cm}$ starting $8 \mathrm{~cm}$ from the end of the stem. During delay testing, 
the ends of the stem and hardware cloth coincided, providing $28 \mathrm{~cm}$ of neutral gray smooth floor where the delay was spent behind a gray shield. A gray shield was also placed behind the rat to prevent resampling of stem cues on the longer delay intervals. In the choice point and arm, the hardware cloth floor had the same dimensions and placement as a color insert. Rubber bands that went under the floor held cloth readily and firmly in place. There were no hurdles anywhere, and, because there was no cue shield, zero delay rather than simultaneous MTS was in effect during initial training. Zero delay was actually $1 / 2$ sec or more, the time needed to reach the floor cues of the arms after entering the neutral area.

\section{Procedure}

Preliminary training. This period lasted 6 days. On Days 1-3, the rats were placed 3 at a time on the gray elevated maze with a changing mixture of black and white hurdles. A 97-mg Noyes pellet was located approximately every $25 \mathrm{~cm}$, and 10 pellets were in each food tray. The rats remained there $30 \mathrm{~min}$ or until all food was consumed; pellets accidentally displaced were replaced. On Day 4 and thereafter, each rat was placed individually on the maze at the end of the stem. On Days 5 and 6 , pellets were present only in food trays, and all rats remained on the maze until both trays were empty. Generally, by Day 6 , rats had adapted to the narrow stem and height from the floor and ran unhesitatingly down the alley and across the hurdles.

Training. There were two main groups. Group DO (Rats 1, 2, and 3) received differential outcomes for correct matches to black and white. Group Non-DO (Rats 4, 5, and 6) received the same reinforcer for all correct matches. Rats 1 and 2 received five pellets for matching to white and only one pellet for matching to black. Rat 3 was started five weeks later and received four pellets for matching to black and two pellets for matching to white. All Non-DO rats received three pellets for all matches, making the average magnitude of reinforcement the same for both groups. Rat 6 started with Rat 3.

Acquisition training consisted of 16 trials per day 6 days a week through criterial day. The intertrial interval (ITI), during which the rat was in a wire running cage, was approximately $2 \mathrm{~min}$. Squads of 2 to 4 rats were run in rotation. On 8 intermixed trials the stem area and the movable shield were black, and on 8 they were white. The color schedule for stem and arms varied daily, with the stipulation that no area remained the same color for more than three successive trials. The black/white shield, which matched the color of the stem for the rat, was slowly moved toward the choice point by the experimenter as the rat advanced down the stem, and the shield ended up between the two arms so that the rat still had a sample stimulus to match when confronted with the comparison stimuli. That is, at least in part, simultaneous MTS obtained during initial training. The relatively long stem with hurdles yielded responsedependent exposures to the sample stimulus that were generally comparable to or slightly longer than the typical 5-sec duration of the sample in operant situations. Exposure to comparison stimuli was subjectdetermined and varied from less than $1 \mathrm{sec}$ to $15 \mathrm{sec}$ or so.

Initially, DO Rats 1 and 2 received noncorrection trials. The subjects were retrieved and returned to the ITI cage about $5 \mathrm{sec}$ after an incorrect match and immediately after consuming all reward pellets. After little or no evidence of learning, correction trials were instituted by returning the rat to the end of the stem following an error. When no evidence of learning occurred with a correction procedure, errorless training was instituted by using a clear plastic barrier to force the rat to take the correct arm. Rat 3 received errorless training throughout. When the daily two probe test trials (nonforced) indicated roughly $50 \%$ correct performance and a strong color preference (see Table 1, column 5), rewards to each arm were equated for these rats and training continued with conventional, noncorrection trials until a criterion of $90 \%$ correct on two successive days was reached. The type and number of training trials used for DO subjects are given in Table 1 .

Non-DO Rats 4 and 5 received largely noncorrection trials, but were given a comparable number of correction and errorless trials at about the same points in time as DO Rats 1 and 2. Training was terminated after 704 trials if criterion was not reached. Rat 6 had errorless training throughout.
Table 1

Number and Order of Training Trials and Terminal Performance for DO (Differential Outcome) Subjects in Black/White Matching-to-Sample

\begin{tabular}{lccc}
\hline & \multicolumn{3}{c}{ Subjects } \\
\cline { 2 - 4 } & 1 & 2 & 3 \\
\hline Noncorrection Trials & 172 & 284 & - \\
Correction & 72 & 36 & - \\
Errorless & 110 & 168 & 172 \\
\% Correct to Smaller Reward & 12.5 & 8.3 & 12.5 \\
$\quad \begin{array}{l}\text { All 3 Days Prior to Equation } \\
\text { Noncorrection Trials After }\end{array}$ & 128 & 184 & 164 \\
$\quad$ Rewards Were Equated (No DO) & & & \\
Total Trials to Criterion & 482 & 672 & 336 \\
Final Performance Level & $90.6 \%$ & $96.9 \%$ & $94 \%$ \\
\hline
\end{tabular}

Delay testing. Delay testing consisted of 32 trials per day, with subjects having access to water midway through the session. There were five delay intervals: $0,2,4,8$, and $12 \mathrm{sec}$. Forty-eight trials were given per delay interval per rat, 24 with each stem color and 24 correct for each arm, delivered in a random, interspersed fashion across days, intervals, stem color, and color of each arm.

All rats received three pellets for each correct match during delay testing. A delay interval was the time between the rat's crossing the second hurdle in the stem and the removal of the gray shield at the choice point by the experimenter. This interval was timed using a well-trained counting procedure. Spot-checking of the experimenter's timing was done repeatedly with a stopwatch. The mean interval values obtained were $2.2,4.1,8.0$, and $12.0 \mathrm{sec}$. For zero delays, the shield was omitted altogether.

Test for transfer. At the end of delay testing of the DO animals on the black/white problem, all 6 rats were shifted to hardware cloth/smooth floor MTS. All rats except Rat 4 were given conventional Non-DO trials with three pellets per reinforcement. Rat 4 , under DO procedure, received four pellets for matching hardware cloth and two pellets for matching smooth floor. Criterion was $87.5 \%$ correct on 1 day.

\section{RESULTS}

There was clearly a difference between groups in MTS accuracy on the black/white problem. All 3 DO rats showed improved performance soon after outcomes for each match were equated, and they subsequently reached acquisition criterion in a mean of 158.6 trials (see Table 1). Their delay performance is depicted individually in Figures 1a-1c and combined in Figure 1d. Rats 4 and 5 were $61 \%$ and $65 \%$ correct, respectively, on the last 3 days of training (Trials 656-704) and had approached that level for many days. Non-DO Rat 6 , given errorless training, reached a criterion of $90.6 \%$ correct in 448 trials, but its performance disintegrated when a zero delay was introduced. Thus, delay testing was precluded for all NonDO subjects.

On transfer to the hardware cloth/smooth floor problem, Rats 2,3,5, and 6 still hovered aroung $50 \%$ performance after 352 noncorrection trials and were discontinued. Rat 1 showed virtually immediate transfer to stable correct performance. The criterion was attained in 22 trials including criterial trials. Rat 4 , the only subject trained on this problem starting with the DO procedure, received 224 trials before outcomes were equated, and then went 
(a)

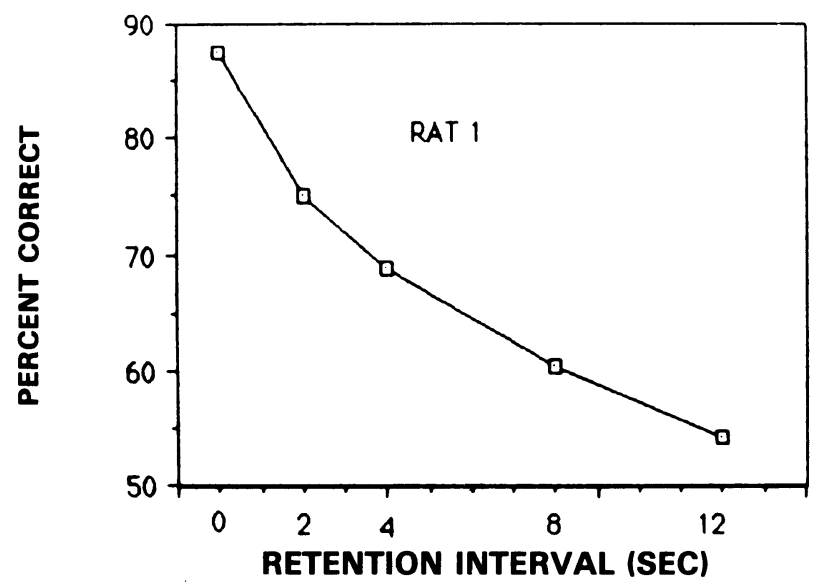

(c)

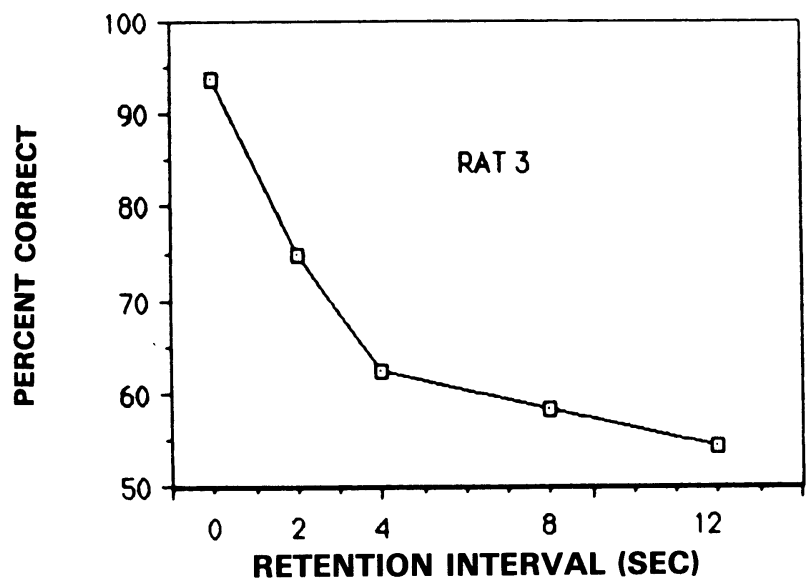

(b)

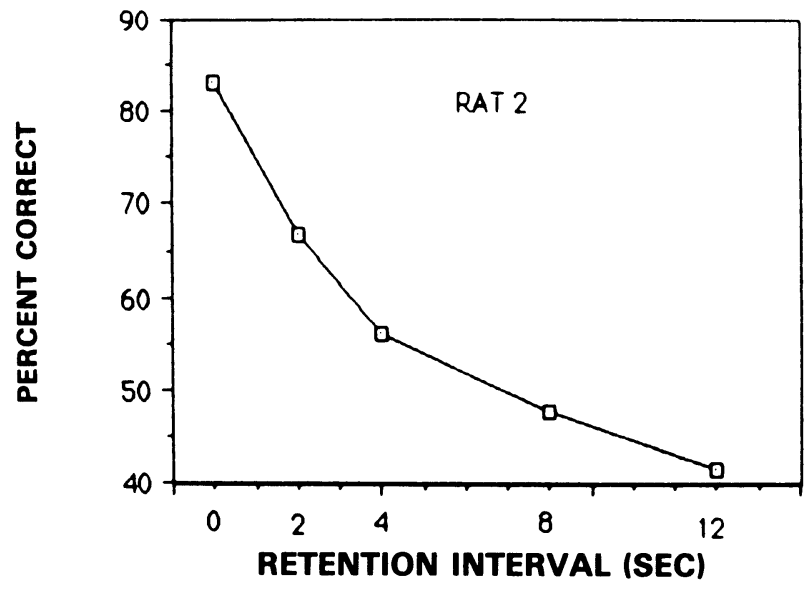

(d)

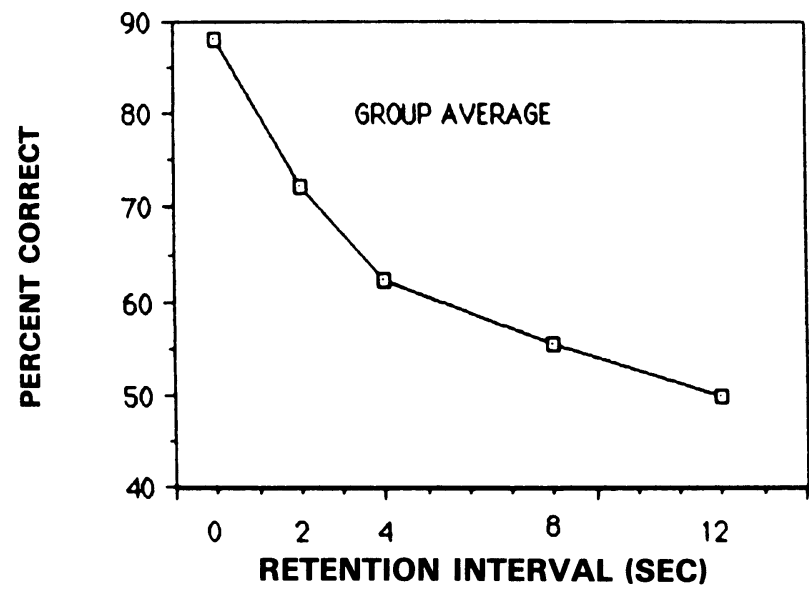

Figure 1. Percent correct for each delay interval on the black/white problem for (a) Rat 1, (b) Rat 2, (c) Rat 3, and (d) the group average.

on to attain criterion in 160 equation trials. Like Rat 1 , Rat 4 performed satisfactorily on delay trials for the hardware cloth/smooth floor problem.

Finally, transfer was tried with Rat 4 by representing the black/white problem. Transfer was almost immediate. In 24 trials, including criterial trials, Rat 4 hit $90 \%$ correct and maintained that level the following day. On delay testing on the black/white problem, performance of Rat 4 (Figure 2) was very similar to that of other subjects, even though it had very few black/white trials with the transfer procedure.

Rats 1, 2, and 3 learned the black/white problem, but only Rat 1 showed transfer to the hardware cloth/smooth floor problem. The only other learner was Rat 4 on hardware cloth/smooth floor MTS. It showed immediate transfer to the black/white problem. Thus, half the subjects tested for transfer showed it decisively, and the other half not at all. And the 2 that showed it did so in opposite directions.

\section{DISCUSSION}

It seems clear that the rats' delayed MTS performance is good and stable and roughly comparable to that of the pigeon (Carter \& Werner, 1978). In fact, the short-term memory of adult humans for visual stimuli is not appreciably superior (L. R. Peterson \& M. J. Peterson, 1959).

Needless to say, the transfer results and the beneficial effect of switching from a DO procedure to equated rewards require more research before their actual status can be determined. The present findings provide only hints in these directions. Nevertheless, even monkeys take well over a thousand trials on average to learn MTS (D'Amato \& Colombo, 1985; D'Amato, Salmon, \& Colombo, 1985), and rats and pigeons in more conventional operant situations take even longer (Wallace et al., 1980; Zentall \& Hogan, 1978). Thus, there is a sizable comparison group for the 4 rats that learned in a mean of 469.5 trials by means of the switching maneuver.

The idea of switching originated as the training trials were being run. It was obvious that MTS learning requires the rat to attend to both relevant cues. It also became obvious that our DO procedure forced the rat to focus on the relevant cue dimension, but that the rat would persist in avoiding the color cue associated with the smaller reward ad infinitum if the DO procedure were maintained (an untested inference, but one based on 47 years of running rats). In any case, here was an 


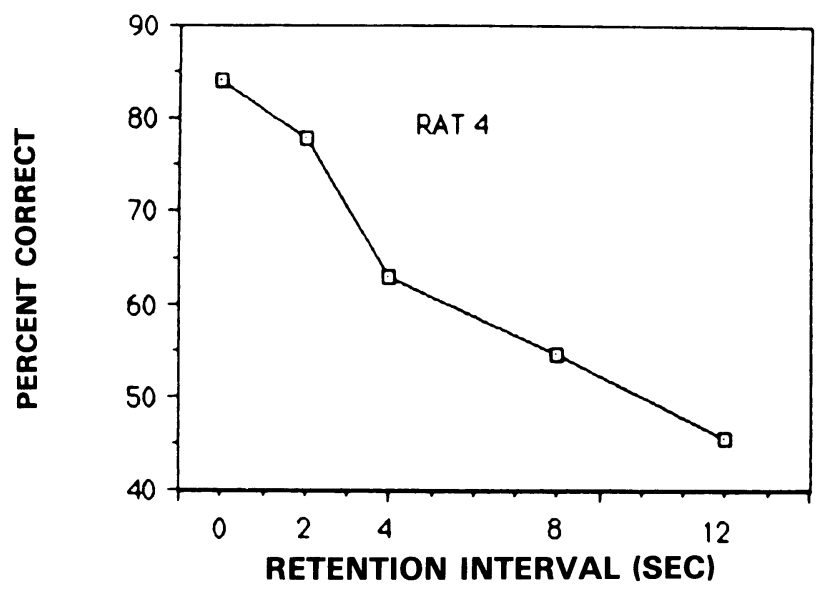

Figure 2. Percent correct for each delay interval on the black/white transfer problem for Rat 4.

ideal opportunity to facilitate the learning of MTS by eliminating the cue-bias while the rat was still attending to the appropriate cues. When the bias was eliminated by equating outcomes for each match, the learning of MTS did indeed occur fairly rapidly.

Future research might determine whether unswitched rats that are maintained on the present DO procedure ever learn MTS. It would seem most unlikely.

The evidence for any sort of transfer effect in pigeons is minimal (Carter \& Werner, 1978; Wilson, Mackintosh, \& Boakes, 1985a; Zentall \& Hogan, 1978), although the European jay has shown good transfer from line to color (Wilson, Mackintosh, \& Boakes, 1985b). Nothing like extradimensional immediate transfer has been reported except in adult and infant chimpanzees (Nissen, Blum, \& Blum, 1948; Oden, Thompson, \& Premack, 1988). To our knowledge, the current study is the first to find any sort of MTS transfer in rats. That only half the tested rats showed transfer indicates the prevalence of individual differences in complex tasks, as also found by Lashley (1938) in conditional discrimination learning.

It is, of course, possible that spontaneous transfer by Rat 4 was helped by the original 704 Non-DO trials on the black/white problem, but this was not the case for Rat 1 . Moreover, the behavior of Rat 4 argues against the original, conventional training's being an important factor in the transfer effect. Once Rat 4 was near criterion on hardware cloth/smooth floor MTS, it would on occasion resample the stem stimuli or correct an incipient wrong response before crossing the arm-hurdle. These sorts of behavior carried over to the black/white transfer problem, but had never occurred during the original black/white trials.

The observed transfer is not exclusively crossmodal. The hardware cloth, of course, provided an extradimensional visual component (texture but not brightness) that distinguished it from the smooth floor. However, the rats that learned indicated from their behavior that the tactual component was critical. When performing well, they frequently stopped at the start of the hardware cloth arm and explored it with their forepaws before appropriately advancing or retreating.

Since even ordinary conditional discriminations that are facilitated by differential outcomes require close to 1,000 trials to learn (Trapold, 1970), the maneuver used here to equate outcomes after the rat has clearly attended to the appropriate cues would seem especially efficacious if the present results can be replicated.

\section{REFERENCES}

Carter, D. E., \& Werner, T. J. (1978). Complex learning and information processing by pigeons: A critical analysis. Journal of the Experimental Analysis of Behavior, 29, 565-601.

Cohen, J. S., EscotT, M., \& Ricciardi, P. (1984). The role of reinforcement symmetry and stimulus modality in successive delayed matching to sample in the rat. Canadian Journal of Psychology, 38, 63-79.

Cohen, J. S., Galgan, R., \& Fuerst, D. (1986). Retrospective and prospective short-term memory in delayed response tasks in rats. Animal Learning \& Behavior, 14, 38-50.

D'Aмато, M. R., \& Соцомво, M. (1985). Auditory matching-to-sample in monkeys (Cebus apella). Animal Learning \& Behavior, 13, 375-382.

D’Amato, M. R., Salmon, D. P., \& Colombo, M. (1985). Extent and limits of the matching concept in monkeys (Cebus apella). Journal of Experimental Psychology: Animal Behavior Processes, 11, 35-51.

HeISE, G. A., \& HuDSON, J. D. (1985). Effects of pesticides and drugs on working memory in rats: Continuous non-match. Pharmacology Biochemistry \& Behavior, 23, 599-605.

Lashley, K. S. (1938). The mechanism of vision: XV. Preliminary studies of the rat's capacity for detail vision. Journal of General Psychology, 18, 123-193 (pp. 175-176).

Nissen, H. W., Blum, J. S., \& Blum, R. A. (1948). Analysis of matching behavior in chimpanzee. Journal of Comparative \& Physiological Psychology, 41, 62-74.

Oden, D. L., Thompson, R. K. R., \& Premack, D. (1988). Spontaneous transfer of matching by infant chimpanzees (Pan troglodytes). Journal of Experimental Psychology: Animal Behavior Processes, 14, 140-145.

Peterson, G. B., \& Trapold, M. A. (1980). Effects of altering outcome expectancies on pigeon's delayed conditional discrimination performance. Learning \& Motivation, 11, 267-288.

Peterson, L. R., \& Peterson, M. J. (1959). Short-term retention of individual verbal items. Journal of Experimental Psychology, 58, 193-198.

Spencer, D. G., JR., Pontecorvo, M. J., \& Heise, G. A. (1985). Central cholinergic involvement in working memory: Effects of scopolamine on continuous nonmatching and discrimination performance in the rat. Behavioral Neuroscience, 99, 1049-1065.

Trapold, M. A. (1970). Are expectancies based upon different positive reinforcing events discriminably different? Learning \& Motivation, 1, 129-140.

Wallace, J., Steinert, P. A., Scobie, S. R., \& SPEar, N. E. (1980). Stimulus modality and short-term memory in rats. Animal Learning \& Behavior, 8, 10-16.

Wilson, B., Mackintosh, N. J., \& Boakes, R. A. (1985a). Matching and oddity learning in the pigeon: Transfer effects and the absence of relational learning. Quarterly Journal of Experimental Psychology, 37B, 295-311.

Wilson, B., Mackintosh, N. J., \& Boakes, R. A. (1985b). Transfer of relational rules in matching and oddity learning by pigeons and corvids. Quarterly Journal of Experimental Psychology, 37B, 313-332.

ZeNTALl, T. R., \& HogAN, D. E. (1978). Same/different concept learning in the pigeon: The effect of negative instances and prior adaptation to transfer stimuli. Journal of the Experimental Analysis of Behavior, 30, 177-186.

(Manuscript received May 20, 1988.) 